\title{
Modification of Maximal Ratio Combining Technique for Detection of Spectrum Hole in a Cognitive Radio Network
}

\author{
Abolade, R.O ${ }^{\mathrm{a}}$, Ojo, S.I ${ }^{\mathrm{b}}$, Ojerinde, I.A ${ }^{\mathrm{c}}$, Adetunji, J.S ${ }^{\mathrm{d}}$, Lawal, A.T ${ }^{\mathrm{e}}$ \\ (a,b,c,d,e) Electronic and Electrical Engineering Department, Ladoke Akintola University of Technology, \\ Ogbomoso, Oyo State, Nigeria
}

Received: 08 January 2020; Accepted: 08 March 2020; Published: 08 April 2020

\begin{abstract}
Spectrum Sensing (SS) is a critical operation in a Cognitive Radio (CR) network to identify spectrum hole thereby preventing licensed users from harmful interference for improving spectrum utilization. However, multipath effects in wireless channel such as multipath fading, shadowing and receiver uncertainty affect the sensing accuracy of CR resulting to high Probability of Missing (PM) that causes interference to Primary User (PU). Maximal Ratio Combining (MRC) technique which was one of the techniques used to solve this problem suffers hardware complexity resulting in high Sensing Time (ST). Therefore, in this paper, modification of MRC technique is carried out to reduce hardware complexity of conventional MRC thereby reducing ST. The modified technique consists of ' $\mathrm{L}$ ' Secondary User (SU) antennas that received the multiple copies of Primary User (PU) signals over Nakagami-m fading channel. The received PU signals are made to passed through separate channel estimator and co-phased to avoid signal cancellation before been summed up. The resultant signa is then made to passed through single RF chain and MF. Output of MF is then used as input to Energy Detector (ED) to obtain the energy of the received signal. The obtained energy is compared with the set threshold to determine the status of spectrum. The modified MRC technique is incorporated with simulation model which consists of PU transmitter that processes the randomly generated data through some signal processing techniques for transmission. Mathematical expression of Probability of False Alarm (PFA) for the modified MRC technique is derived and used to set the thresholds at PFA of 0.01 and 0.05 . The modified model is evaluated using PM, Probability of Detection (PD) and PT to determine the performance. The results obtained revealed that modified MRC gives higher PD, lower PM and PT values when compared with conventional MRC.
\end{abstract}

Index Terms: Maximal Ratio Combiner, Energy Detector, Probability of False Alarm, PD, PM, PT, Cognitive Radio.

(C) 2020 Published by MECS Publisher. Selection and/or peer review under responsibility of the Research Association of Modern Education and Computer Science

\footnotetext{
*Corresponding author. Tel.: +2348167429962
}

E-mail address: siojo85@pgschool.lautech.edu.ng 


\section{Introduction}

The transfer of information between two or more points that are not physically connected known as wireless communication, is experiencing a tremendous growth due to rapid increase in the number of users accessing its services. The rapid growth of wireless communication resulting in scarcity of radio spectrum. Past researches on spectrum revealed that, the scarcity of radio spectrum is majorly due to underutilization of allocated spectrum. While certain spectral ranges such as mobile communication bands are constantly clustered, there are large band of spectrum where average usage is below 10\% [1]. The problem of spectrum scarcity is overcome by exploiting unused spectrum spaces through dynamic spectrum access using Cognitive Radio (CR). CR is a form of wireless communication in which a transceiver intelligently detects the radio spectrum in use and the one not in use. After which it is then instantly moved into idle spectrum while avoiding occupied ones [2, 3, 4]. The radio comprises of two users namely: Primary User (PU) and Secondary User (SU). PU is the licensed user that has privileges to the assigned spectrum and $\mathrm{SU}$ is the unlicensed user which makes use of radio spectrum only when PU is idle [5].

The radio consists of four core operations namely: Spectrum Sensing (SS), Spectrum Analysis (SA), Spectrum Decision (SD) and Data Transmission (DT). SS is a phenomenon in which SU senses the radio spectrum to detect the presence of PU and thereby identifies empty spectrum spaces known as spectrum hole [6, 7]. Detection of spectrum hole (empty spectrum) is of paramount important in CR network for SU to transmit through licensed spectrum without interfering with any licensed user. PU detection happened to be the most efficient way to detect empty spectrum [8]. The integrity of CR relies on the ability of the SU to restrict interference to PU and maintain a reliable quality of service for its own transmission [6, 9, 10]. Spectrum analysis is the process by which SU analyses the detected spectrum hole and determines whether the hole is useful for the desired SU transmission. Spectrum decision decides on which empty spectrum to continue with transmission and considers all the PU nodes within the SU network. Decision which is the instruction from SS, may instruct the nodes to remain on the current spectrum, if PU is not active and immediately moves to the next backup spectrum if PU is present. Data transmission is a process by which SU transmits the data through the empty spectrum and adapts the transmission parameters such as frequency and modulation scheme to suit the propagation characteristics of different PU spectrum [6].

Accuracy of SS which is the ability of SU to sense PU without interference, depends on multipath propagation. Multipath propagation is a phenomenon that occurs when a transmitted signal propagates in multiple copies as a result of obstructions along propagating path. Multipath propagation resulting in fluctuation of the PU signals which could be so severed as to produce a low signal below the sensitivity of the SU and this causes poor reception of PU signal thereby making PU difficult to sense [22]. One of the challenges encountered in CR network is hidden PU problem which resulted from difficulty in sensing PU signals. Hidden PU problem caused interference in CR due to difficulty in detecting presence of license user. Therefore, hidden PU in CR network needs to be mitigated. Several techniques such as Selection Combiner (SC), Maximal Ratio Combiner (MRC) and Equal Gain Combiner (EGC) has been used to combat the effect of hidden PU in CR system. MRC showed better performance when compared with SC and EGC but at the expense of hardware complexity [11, 20]. According to [22, 21], the complexity of MRC is due to multiple Radio Frequency (RF) chain and Match Filter (MF) used in the technique. Therefore, in this paper a modified MRC using single RF chain and MF to reduce the hardware complexity of the existing MRC thereby reducing sensing time is developed.

\section{Related Work}

[18] presented a paper on multiple detectors based analytical performance of spectrum sensing to solve problem of interference in CR system using Maximal Ratio Combining technique. In their work, Secondary 
User (SU) received data from each antenna and multiplies them with the conjugate of each channel gain. The multiplied data was then summed and applied to only one Energy Detector (ED). Output of ED was then compared with the decision threshold to make decision on whether spectrum is busy or idle. The result of this work showed that, the technique has a better performance by having a high Probability of Detection (PD) but suffer from hardware complexity due to knowledge of the instantaneous PU signal at each branch. Also, [20] worked on distance based an efficient transmit power control scheme in CR system with multiple antennas. PU interference in CR was addressed using Selection Combining (SC) technique. In this paper, PU select the branch with the highest channel gain and received data from that antenna. The received data serves as input to ED to make a final decision on whether PU signal is present or not. One of the most promising advantages of this method was the fact that, the threshold does not change as the noise level remains constant. The result of this paper showed that, the technique has a very low hardware complexity but at the expense of poor detection rate when compared to MRC. The existing works suffer from poor detection rate resulting in high interference when SC is used and MRC developed to solve the problem of SC suffers from hardware complexity resulting in high sensing time. Due to these shortcomings, this paper therefore, develops a modified MRC using single RF chain and MF to reduce sensing time and thereby improving performance of the system.

\section{Materials and Method}

The material used in this work include Energy Detector (ED), Maximal Ratio Combiner (MRC) and Binary Phase Shifting Keying (BPSK) modulation scheme. The existing MRC technique by [18] is modified by replacing multiple RF chain and MF with single RF chain and MF. Closed form expression of Probability of False Alarm (PFA) for the modified model is derived and used to set decision threshold. Simulations using Matrix Laboratory simulation software is used to investigate the performance of the modified technique. Performance of the modified technique is evaluated using Probability of missing (PM), Probability of Detection (PD) and Processing Time (ST) by comparing with the conventional MRC.

\subsection{Energy Detector (ED)}

Energy Detector (ED) is a detection technique used to detect the presence or absence of signal in the band using energy of the received signal. ED is a commonly used in spectrum sensing due to its low computational complexities and it can be implemented in both time and frequency domain. Signal detection using ED is carried out by comparing the output of ED with decision threshold which depends on the noise floor of the fading channel. The detector is based on two assumptions namely; the noise must be statistically stationary and variance known to the detector [1]. ED simply needs a Band-Pass Filter (BPF) which allows frequencies within a certain range and attenuates frequency outside that range, an analog to digital converter, square law device and an integrator $[11,13]$. The block diagram of ED is shown in Fig. 1. The input signal $q(t)$ is filtered with a BPF to select the specific band of frequency to which SU wants to sense. This signal is squared by a squaring device to obtain the energy of the received signal and output of squaring device is passed through integrator to determine the observation interval. The output of the integrator is compared with the predetermined threshold. If the values are above the threshold, then, PU is present otherwise PU is absent [14]. ED has advantage of implementing without any prior knowledge of PU signal compared to other detector such as match filter and cyclostationary. This advantage makes it easy to set decision threshold when using ED [14]. Output of energy detection, " $E_{t i m e}$ " is given by [15] as

$$
E_{\text {time }}=\int_{n}^{N}(q(n))^{2}
$$

where:

$\mathrm{N}$ is the symbol length to be sensed and 
$q(n)$ is the transmitted signal by Primary User.

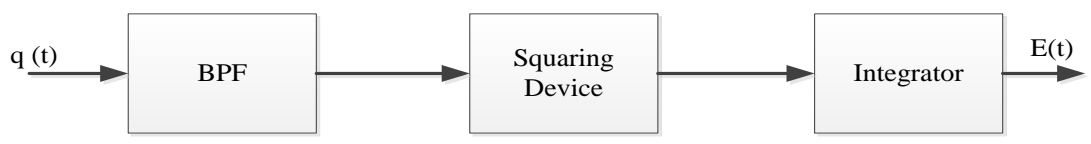

Fig.1. Block diagram of Energy detector

\subsection{Maximal Ratio Combiner (MRC)}

Maximal Ratio Combiner (MRC) is a diversity combining technique used in CR network to improve detection rate. MRC is a technique in which all the branches of the signals are made to passed through channel estimator to determine the weight of each branch and multiplied with the signal on individual branch. Signal output of channel estimator is then co-phased to avoid signal cancellation before summing, to provide the optimal SNR at the output. In MRC, the weights are chosen as proportional to the respective signals level for maximizing the combined Carrier-to-Noise Ratio (CNR). MRC is the optimal combining scheme (regardless of fading statistics) but at the expense of hardware complexity due to multiple RF chain and MF involved (Ramanathan, 2003). The received signal passes through RF chain, MF and then, sums all the signals before applying Energy Detector (ED) as shown in Fig. $2[17,9]$. The output signal of MRC with ED ' $E_{M R C}$ ' is given by [9] as

$$
E_{M R C}=\sum_{K=1}^{N}\left|\left(\sum_{j=1}^{N_{r}} a_{j} q_{j}(k)\right)\right|^{2}
$$

where:

$\mathrm{N}$ is the symbol length

$N_{r}$ is the number of antenna,

$q_{j}(k)$ is the transmitted signal by $\mathrm{PU}$

$a_{j}(k)$ is the channel gain on individual branch

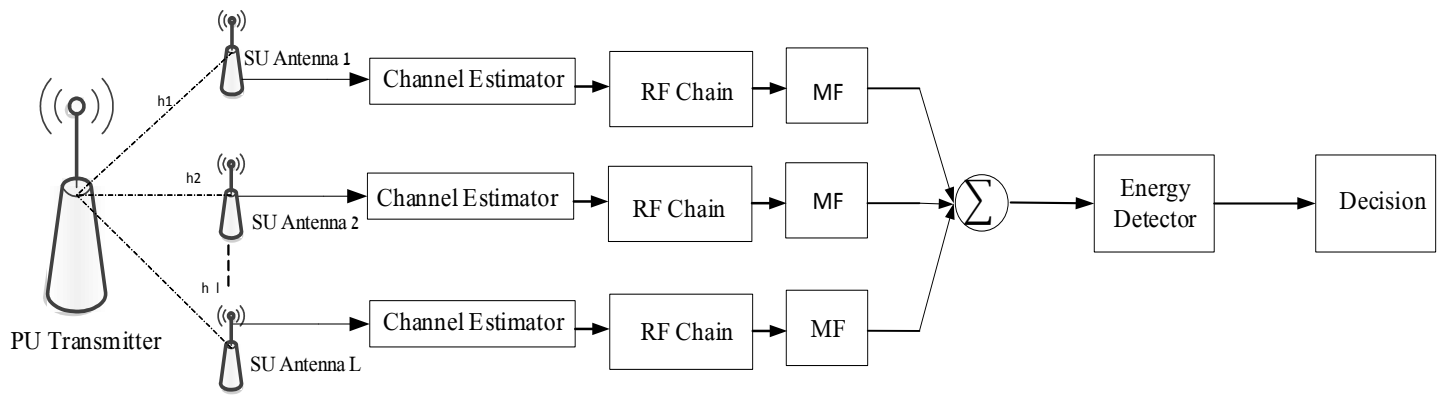

Fig. 2. Conventional MRC with Energy Detector Source: [18]

\subsection{Binary Phase Shift Keying (BPSK)}

Binary Phase Shift Keying (BPSK) is a digital modulation technique in which the phase of carrier signal varies in accordance with the phase of baseband signal. It is one of the simplest forms of phase modulation that conveys data by changing the phase of a reference signal. The phase of a constant amplitude carrier signal moves between 0 and $180^{\circ}$ as shown in Fig. 3. There are two possible locations in the state diagram in which 
binary one or zero is transmitted. The symbol rate of this type of modulation is one bit per symbol [19]. The general expression for BPSK signaling scheme ' $S_{n}(t)$ ' is given by [19] as

$$
S_{n}(t)=\left(\frac{2 A_{b}}{T}\right)^{\frac{1}{2}} \cos \left(2 \pi f_{c} t+\pi(1-n)\right)
$$

where:

$A_{b}$ is amplitude of base signal

$T$ is bit duration

$f_{c}$ is the carrier frequency

$n=0,1$

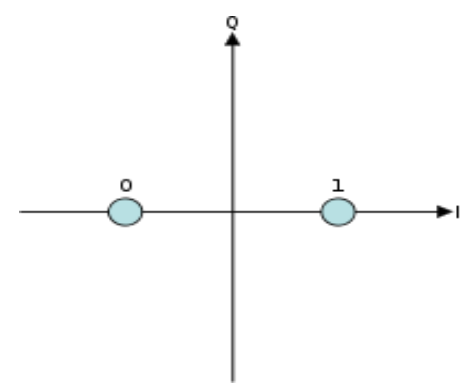

Fig. 3. Constellation diagram for BPSK

\subsection{Modification of Maximal Ratio Combiner}

Conventional Maximal Ratio Combiner (MRC) technique is modified by replacing several RF chain and MF with only one RF chain and MF as shown in Fig 4. In this modified technique, the PU signal transmitted over Nakagami-m fading channel is received by ' $\mathrm{L}$ ' SU antennas. The received SU signals is made to passed through channel separate channel estimator to determine the channel gain of individual branch. The estimated channel gain is then multiplied with the received signal. The output signals from individual channel estimator are co-phase to avoid signal cancellation and summed up before passed through single RF chain and MF. $h_{1}$, $h_{2}$ and $h_{L}$ represent Nakagami-m fading channels. Signal output of MF is then used as input to only one ED to obtain the energy of the received signal and compared with the set threshold at PFA of 0.01 and 0.05 to make decision on the presence or absence of PU signal. If the obtained energy is greater than the set threshold, then spectrum is busy, otherwise spectrum is idle.

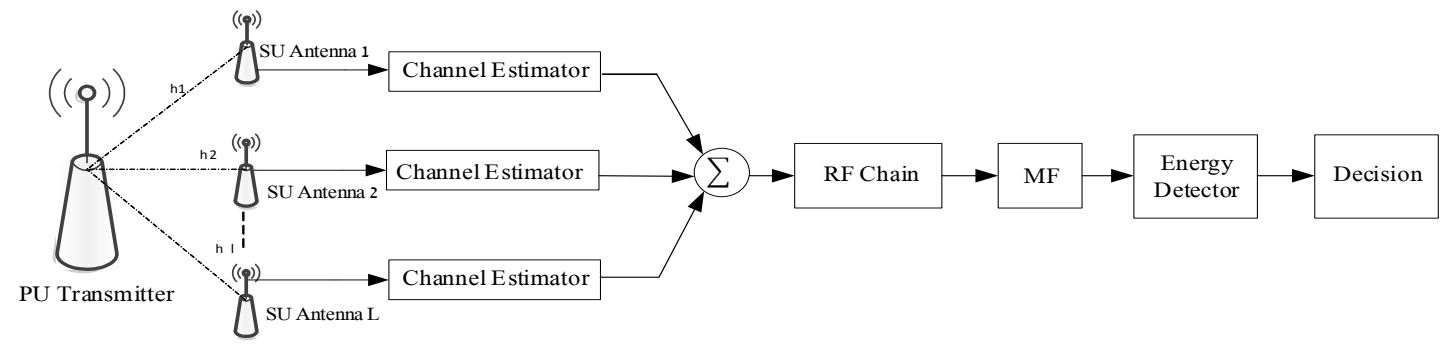

Fig. 4. Modified MRC with Energy Detector 
Output of ED for the modified MRC is obtained as

$$
E_{m M R C}=\sum_{n=1}^{N}\left|\frac{1}{w L}\left(\sum_{i=1}^{L} H \times S(i)\right)^{2}\right|^{2}
$$

where:

$\mathrm{N}$ is the symbol length

$L$ is the number of antenna,

$S(i)$ is the PU signal

$\mathrm{H}$ is the channel gain on individual branch

$\mathrm{w}$ is the noise present

SU then uses threshold to make decision on the presence or absence of PU signal to determine the status of licensed spectrum and this threshold is given as

$$
E_{m M R C}>\lambda
$$

where:

$\lambda$ is the decision threshold which depend on PFA

\subsection{Probability of False Alarm}

Probability of False Alarm (PFA) is the probability that, SU decide that spectrum is occupied and spectrum is actually idle. PFA for the modified model is derived as follow. From Equation (4) under $H_{0}$ hypothesis, output of ED for the modified technique ' $E_{m M R C / H_{0}}$ ' is given as

$$
E_{m M R C / H_{0}}=\sum_{n=1}^{N}\left|\frac{1}{w L}\left(\sum_{i=1}^{L} H \times w(i)\right)^{2}\right|^{2}
$$

Using chi-square distribution, Equation (6) becomes

$$
f_{m M R C / H_{0}}(\xi)=\frac{1}{\left(\sum_{n=1}^{N} \frac{1}{w L} \sum_{i=1}^{L} H \times w(i)\right)^{\frac{N}{2}} 2^{N} / 2^{\mathrm{I}(N / 2)}} \xi^{(N / 2)-1} \exp \left(-\frac{\xi}{2 \sum_{n=1}^{N} \sum_{i=1}^{L} H \times w(i)}\right)
$$

To obtain PFA, Equation (7) is integrated with respect to the degree of freedom ' $\xi$ '

$$
P F A_{m M R C}=\int_{\frac{1}{2 \sum_{n=1}^{N} \frac{1}{w L} \sum_{i=1}^{L} H \times w(i)}}^{\infty} f_{M R C / H_{0}}(\xi) d \xi
$$

By integrating Equation (8) with respect to $\xi$ using change of variable, gives

$$
P F A_{m M R C}=\frac{1}{\Gamma(N / 2)} \int_{\frac{1}{2 \sum_{n=1}^{N} \frac{1}{w L} \sum_{i=1}^{L} H \times w(i)}}^{\infty} t^{(N / 2)-1} \exp (-t) d t
$$

Using incomplete gamma function, Equation (9) becomes 


$$
P F A_{M R C}=\frac{\Gamma\left(\frac{\lambda}{2 \sum_{n=1}^{N} \frac{1}{w L} \sum_{i=1}^{L} H \times w(i)}, N / 2\right)}{\Gamma(N / 2)}
$$

where:

$\lceil$ is the gamma function

The threshold is then set at PFA of 0.01 and 0.05

\section{Simulation Model}

The system simulation model for the modified technique consists of PU transmitter, the Nakagami-m fading channel ' $h$ ' and the SU receiver. Data acquisition is generated from the random integer generator, which is available within the MATLAB R2018a. PU transmitter processes the data generated randomly for transmission, by converting the data into bits, reshaping and modulating with BPSK modulation scheme at different trials. The Square-Root Raised Cosine (SRRC) filter is used at the PU transmitter to reduce the bandwidth of transmitted signal for suitable transmission over Nakagami-m fading channel without losing any information and improving the spectral efficiency. The multiple copies of PU signal are received using multiple SU antennas and the received signal are combined at RF chain using modified MRC. Signal output of the combiner is then demodulated using BPSK demodulator after being gray decoded and used as input to ED to obtained the energy of the received signal. The obtained energy is then compared with the set threshold to determine the channel status. $h_{1}, h_{2}$ and $h_{L}$ in Fig. 5 represent Nakagami-m fading channel over different paths and $w_{1}, w_{2}$ and $w_{L}$ represent the AWGN present in the environment. The decision threshold is set using PFA of 0.01 and 0.05 which is derived using chi-square distribution. The complete simulation model for the modified technique is shown in Fig 5.

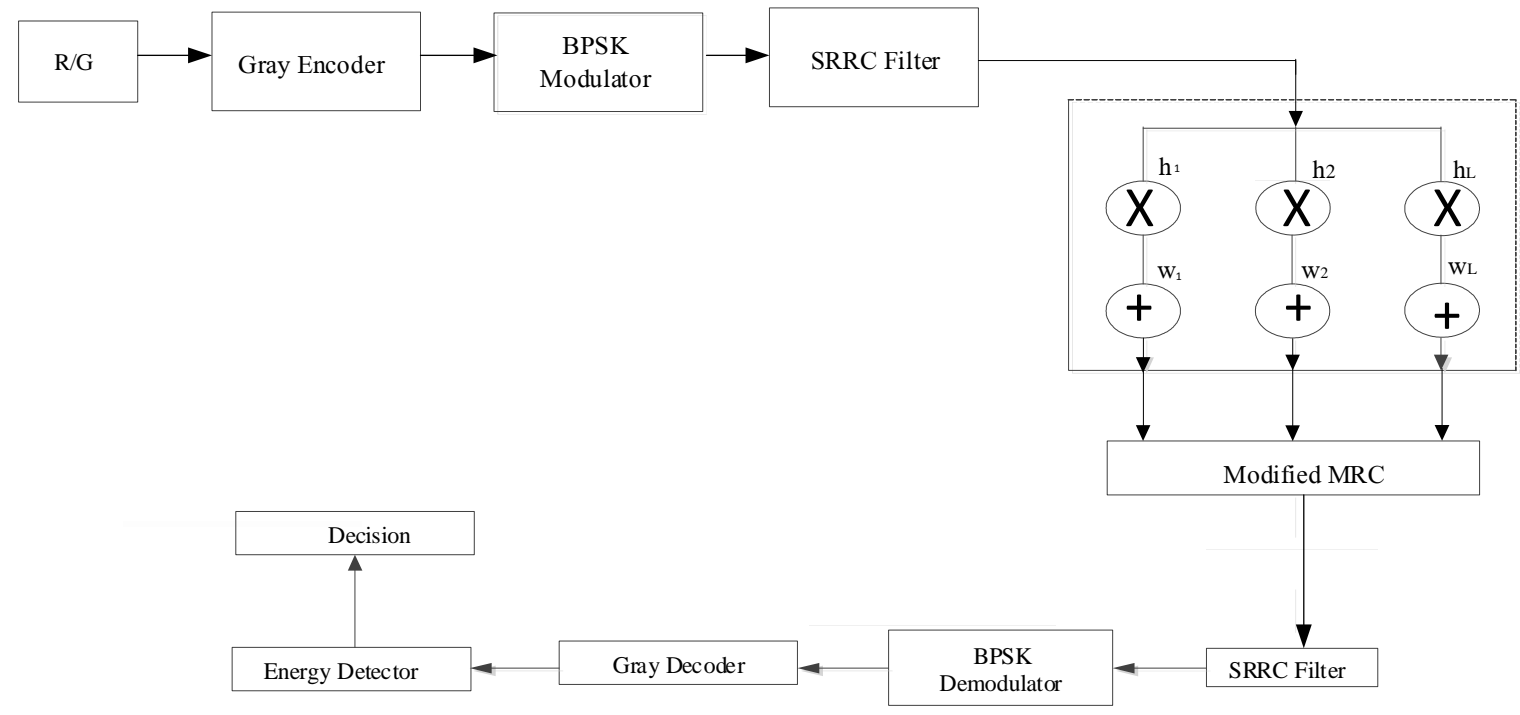

Fig. 5. Simulation model for the modified technique. 


\section{Results and Discussion}

The values of PD, PM and PT obtained for both conventional and modified MRC are plotted against SNR as presented in Figs 6 to 8 at PFA of 0.01. PD values obtained at different SNR are presented in Fig. 6 for both modified MRC and conventional MRC at PFA of 0.01. The PD values obtained for the modified MRC are 0.8387, 0.8585, 0.8776 at SNR of 4, 6, $8 \mathrm{~dB}$, respectively as against the conventional MRC with PD values of $0.7624,0.7805,0.7978$ at SNR of $4,6,8 \mathrm{~dB}$, respectively. Results obtained revealed that, modified MRC showed better performance with high detection rate when compared with conventional MRC due to losses incurred as the signal is passing through multiple RF chain and MF in conventional MRC. This loss is reduced in modified MRC due to single RF chain and MF used. The Probability of Missing (PM) at PFA of 0.01 for both modified and conventional MRC is showed in Fig 7. PM values obtained for modified MRC are 0.1613,

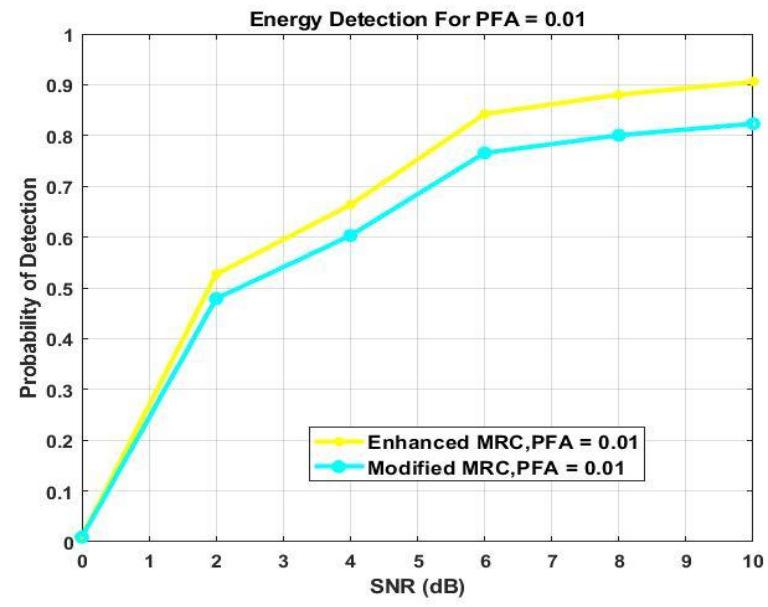

Fig.6. Probability of Detection versus SNR for conventional and modified MRC at PFA of 0.01 .

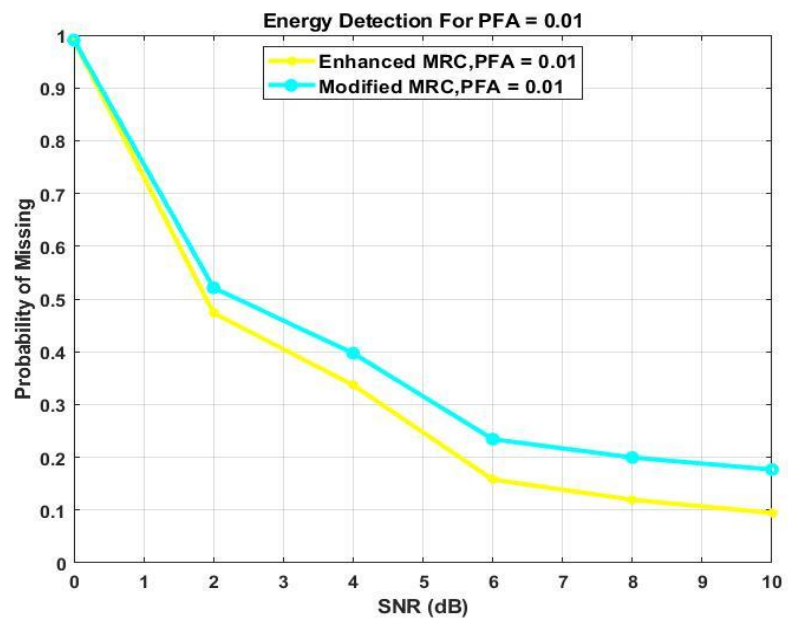

Fig.7. Probability of missing versus SNR for conventional and modified MRC at PFA of 0.01 . 
$0.1415,0.1224$ at SNR of 4, 6, 8 as against $0.2376,0.2195,0.2022$ at SNR of 4, 6, $8 \mathrm{~dB}$, respectively. The values of Processing Time (PT) obtained at different SNR for both modified and conventional MRC is presented in Fig. 8. At SNR of 4, 6, $8 \mathrm{~dB}$, PT values of $0.1697,0.2357,0.2961$ are obtained, respectively as against 0.2546, 0.3536, 0.4441 respectively, obtained for conventional MRC. The lower PT values obtained for modified MRC showed reduction in hardware complexity of modified MRC. The lower PT of the modified technique resulted from hardware reduction demonstrate that, the modified technique will make decision on the presence and absence of PU signal which is required in a CR network. A good sensing technique must be able to scan through the spectrum and make decision at a faster rate.

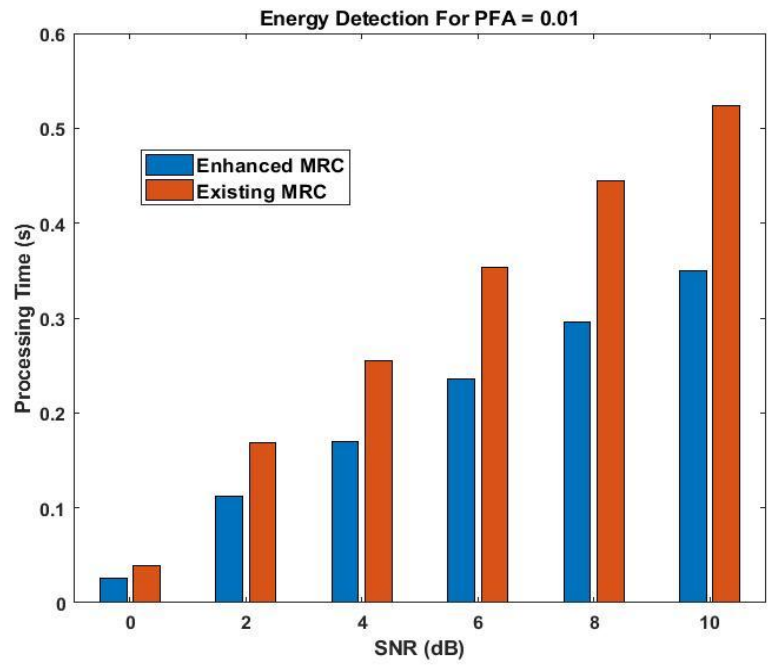

Fig.8. Processing time versus SNR for conventional and modified MRC at PFA of 0.01 .

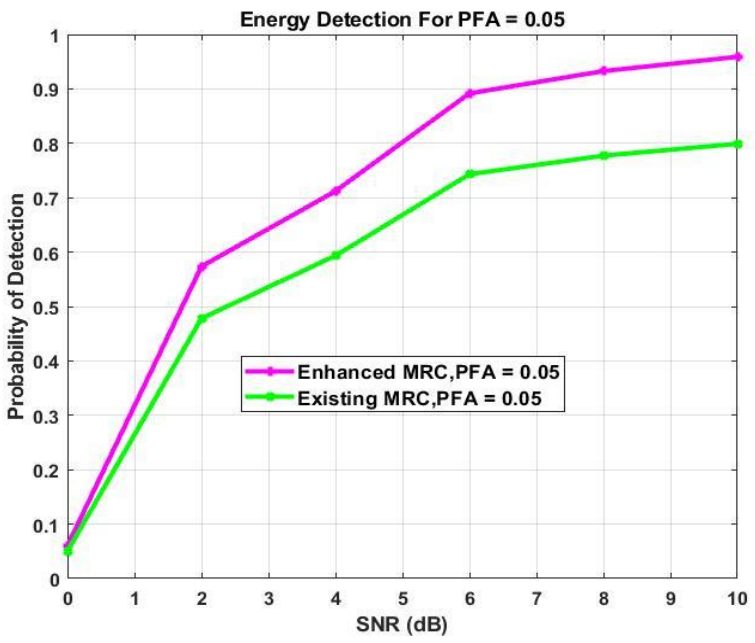

Fig.9. Probability of Detection versus SNR for conventional and modified MRC at PFA of 0.05 . 
Similarly, the PD, PM and PT values obtained at different SNR for PFA of 0.05 are presented in Figs 9 to 11 . Fig 9 depicts the PD obtained at different SNR for both modified and conventional MRC. At SNR of 4, 6, $8 \mathrm{~dB}$, PD values of $0.8863,0.9089,0.9303$ are obtained, respectively, for modified MRC as against $0.7386,0.7574$, 0.7752 obtained for conventional MRC. It can be deduced that, PD values increases as SNR increases and this depict the fact that, ED perform better at high SNR. Also, in Fig 10 PM values of 0.1137, 0.0911, 0.0697 are obtained for modified MRC at SNR of 4, 6, $8 \mathrm{~dB}$, respectively, while $0.2614,0.2426,0.2248$ are respectively obtained for conventional MRC at the same value of SNR. The results obtained revealed that, modified MRC gave better performance with low PM when compared with conventional MRC and this is due to reduction in signal loss due to single RF chain and MF used in the modified technique. PT values obtained at different SNR

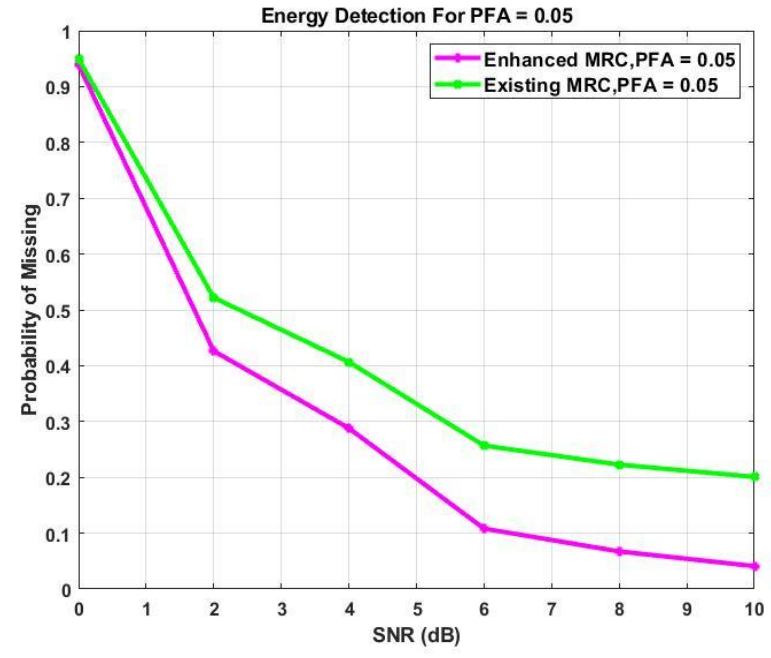

Fig.10. Probability of missing versus SNR for conventional and modified MRC at PFA of 0.05 .

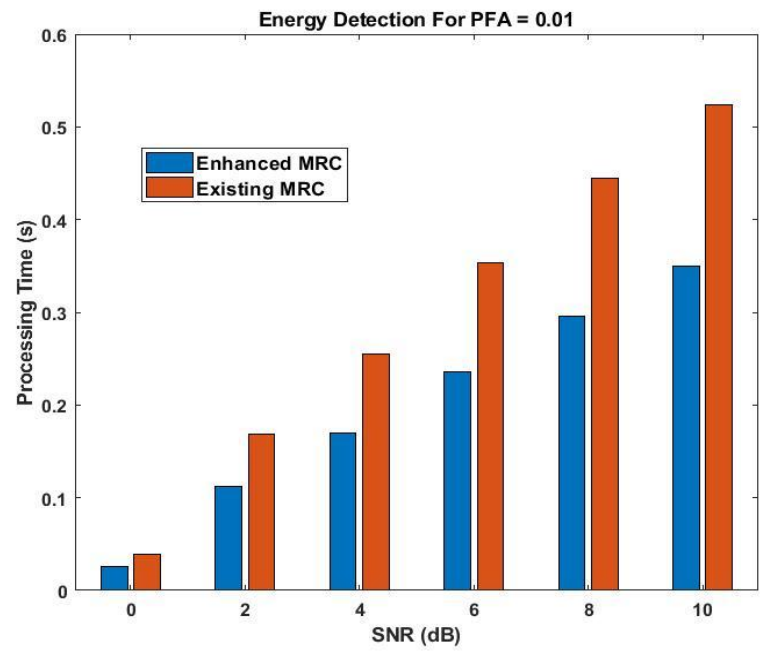

Fig.11. Processing time versus SNR for conventional and modified MRC at PFA of 0.05 . 
for both modified and conventional MRC at PFA of 0.05. are present in Fig 11. At SNR of 4, 6, $8 \mathrm{~dB}$, the PT obtained for modified MRC are $0.1306,0.1813,0.2278 \mathrm{~s}$, respectively, as against $0.1959,0.2720,0.3416$ s,respectively, for conventional MRC. The results obtained are justifiable in that single RF chain and MF used in the modified technique reduces the hardware complexity of modified MRC, thereby reducing the processing time. The results obtained also show that, PD increases as SNR increases and this is due to ED that has poor performance at low SNR. The PD values obtained for both modified and conventional MRC are high at PFA of 0.05 when compared with the PFA of 0.01 . However, higher PFA resulting in inefficient spectrum management.

\section{Conclusions}

Modification of Maximal Ratio Combining (MRC) technique for detection of spectrum hole in a CR network has been developed and simulate in MATLAB environment using appropriate parameters. Closed form expression for Probability of False Alarm (PFA) for the modified technique has been derived using chi-square distribution and used set the threshold at PFA of 0.01 and 0.05 . Performance of the modified technique has been evaluated using PM, PT and PD. The results obtained revealed that, the modified MRC performs better than the conventional MRC with higher PD, lower PT and PM values due to reduction in signal losses resulting from combining nature of the modified MRC technique. Modified MRC gives lower PT values due to single RF chain and MF as against multiple RF chains and MFs used for the conventional MRC. Therefore, the modified MRC has been shown to have a better performance by having a higher PD, lower PM and PT when compared with the conventional MRC. The higher PD and lower PM values of the modified technique shows that it has good detection rate even at a very low SNR and lower PFA value. The lower PT justify the fact that, modified technique can sense the presence of PU signal and make decision as fast as possible which happen to be one of major criterial to be consider in a CR system. The study shows the hardware complexity reduction and increase in detection rate which is suitable for signal detection in a CR network. This work can be improving upon by using the modify technique in a cooperative manner to increase detection rate.

\section{References}

[1] Kevin, C. Spectrum Sensing, Detection and Optimization in Cognitive Radio for Non- Stationary Primary User Signals, unpublished Ph.D Thesis submitted to Queensland University of Technology, Network and Communication, Faculty of Science and Engineering, 2012. pp 12-96.

[2] Ajay, K.G., Raju, U.G., Aravind, P. and Sushma, D. Intelligent Wireless Communication System of Cognitive Radio, International Journal of Emerging Science and Engineering, 2013. 1(4): 78-84.

[3] Mitola J. An Integrated Agent Architecture for Software Defined Radio for Cognitive Radio, PhD thesis submitted to Royal institute of Technology, 2000. pp 10-104.

[4] Hano W. Gosan, N., Dongkyu, K., Sungtae, K. and Daesik, H. Advanced Sensing Techniques of Energy Detection in Cognitive Radio, 2010. Journal of Communications and Networks 12(1): 19-21.

[5] Ojo, F.K. and Fagbola, F.A. Spectrum Sharing in Cognitive Radio Work Using Good put Mathematical Model for Perfect Sensing, Zero Interference and Imperfect Sensing Non-Zero interference, international Journal of Wireless Communication and Mobile Computing, 2015. 3(6): 58-59.

[6] Itilekha, P. and Monami, S. Spectrum sensing in cognitive radio under different fading environment, International Journal of Scientific and Research Publications, 2014. 4(11): 2250-3153.

[7] Mansi, S. and Gajanan, B., Spectrum Sensing Techniques in Cognitive Radio Networks, International Journal of Next Generation Networks, (2011). 3(2): 2011 37-40.

[8] Neelu, A. K. and Shilpi, P. Optimal allocation techniques for reducing the sensing error probability with improved energy detection in Cognitive Radio, International Journals of Advanced Research in Computer Science and Software Engineering, (2017). 7(6):897-899. 
[9] Osama E., Maha E., Osamu M. and Hiroshi, F. Game Theoretic Approaches for Cooperative Spectrum Sensing in Energy-Harvesting Cognitive Radio Networks, IEEE transaction on wireless communication (2018). 28(1):1-5.

[10] Pradeep, K. G., Sachin T. and Rajeshwar, L. D. (2012). Performance analysis of energy detection, matched filter detection and cyclostationary feature detection spectrum sensing techniques, International Journal of Computational Engineering Research 2(5):1296-1297.

[11] Mahmood, A.A. and Zahir, A.H. A new multiple Antennas Method Based Energy Detector for Cognitive Radio over Fading Channels, International Journal of Computer Applications, 2012. 52(5): 20-21.

[12] Komal, P. and Tanuja, D. Review on Spectrum Sensing in Cognitive Radio Using Multiple Antenna, International Journal of Innovative Science Engineering and Technology, 2016. 3(4): 313-315.

[13] Dong, X.J., Chen, Y. B., Yang, G.Y., Pang, X.Z. and Yang, Y. X. The Optimization of Improved Energy Detector in Cognitive Radio Network, International Conference on Computer Information Systems and Industrial Applications, Yunnan Minzu University, 2015. pp 10-107.

[14] Suman, R., Rajeshwar, L. D. and Parmender, S. Spectrum Sensing in Cognitive Radio Using MIMO Techniques, International Journal of Soft Computing and Engineering, 2011. 1(5): 259-261.

[15] Refik, F.U. Spectrum Sensing Techniques for Cognitive Radio Systems with Multiple Antennas, unpublished Master thesis in Electronics and communication Engineering, Graduate School of Engineering and Sciences, 2010. pp 8-134.

[16] Ramanthan V. Performance Evaluation of Equal Gain Diversity Systems in Fading Channel, Master Thesis Submitted to the Faculty of the Virgiria Polytechnic, Institute and State University, 2003. pp 1-4

[17] Rayan, A. H. S., Khalid H. B. and Ibrahim E. Performance evaluation of Energy Detection in spectrum sensing on the Cognitive Radio Networks, Journal of Electrical and Electronic Systems, (2017). 10(4):1-3.

[18] Taruna, T. and Bhumika, P. Multiple Detectors Based Analytical Performance of Spectrum Sensing, International Journal of Advanced Computer Research, 2014. 4 (1), 95-98.

[19] Sushmaja, K. and Fazal, N. Implementation of Binary Shifting Keying Techniques, International Journal of Engineering Trends and Technology, 2013. 4 (6): 2580-2585.

[20] Komal, P. and Tanuja D. (2016). Review on: spectrum sensing in Cognitive Radio using multiple antenna, International Journal of Innovative Science, Engineering and Technology 3(4):313-318.

[21] Adeyemo, Z.K, Ojo, S.I, Abolade, R.O, and Oladimeji, O.B. Modification of a Square-Law Combiner for Detection in a Cognitive Radio Network, International Journal of Wireless and Microwave Technologies, 2019. 9(2): 32-45.

[22] Adeyemo, Z.K. and Ojedokun, I.A. EGC Receiver with Single Radio Frequency Chain and Matched Filter over Combined Rayleigh and Rician Fading Channels, ARPN Journal of Engineering and Applied Sciences, 2014. 9(7): 992-994.

\section{Authors' Profiles}

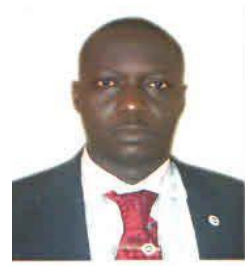

Abolade, Robert $\mathbf{O}$ received His B.Tech. and Ph.D degree in the Department of Electronic
and Electrical Engineering, Ladoke Akintola University of Technology (LAUTECH),
Ogbomoso, Nigeria. He obtained His M.Eng from Department of Electronic and Electrical
Engineering, Obafemi Awolowo University Ile-Ife, Nigeria. He is a registered member of
Council for the Regulation of Engineering in Nigeria (COREN) and member of the IEEE.
His research interest is on signal processing in communications. 


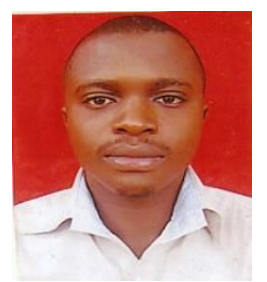

Ojo, Samson I. received his B.Tech and M.Tech degrees from the Department of Electronic and Electrical Engineering, Ladoke Akintola University of Technology (LAUTECH), Ogbomoso, Nigeria in 2011 and 2018, respectively. He is a registered member of Council for the Regulation of Engineering in Nigeria (COREN). Currently his pursuing his Ph.D from the same Department. His research interest is in signal processing and spectrum shearing in wireless communication.

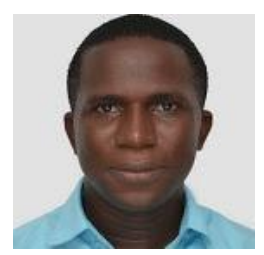

Ojerinde, Idowu A. received his B.Tech from the Department of Electronic and Electrical Engineering, Ladoke Akintola University of Technology (LAUTECH), Ogbomoso, Nigeria in 2014. He is a registered member of Council for the Regulation of Engineering in Nigeria (COREN). Currently his pursuing his M.Tech from the same Department. His research interest is in signal processing in wireless communication.

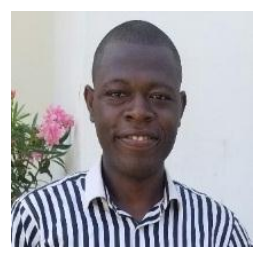

Adetunji, Jeremiah S. received his B.Tech from the Department of Electronic and Electrical Engineering, Ladoke Akintola University of Technology (LAUTECH), Ogbomoso, Nigeria in 2018. He is a graduate member of Nigeria Society of Engineer (GNSE). His research interest is in signal processing in wireless communication.

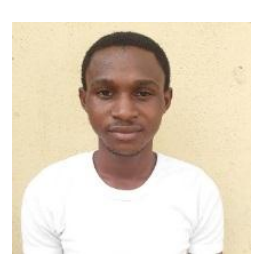

Lawal, Ayodele T. received his B.Tech from the Department of Electronic and Electrical Engineering, Ladoke Akintola University of Technology (LAUTECH), Ogbomoso, Nigeria in 2018. He is a graduate member of Nigeria Society of Engineer (GNSE). His research interest is in signal processing in wireless communication.

How to cite this paper: Abolade, R.O, Ojo, S.I, Ojerinde, I.A, Adetunji, J.S, Lawal, A.T, " Modification of Maximal Ratio Combining Technique for Detection of Spectrum Hole in a Cognitive Radio Network ", International Journal of Wireless and Microwave Technologies(IJWMT), Vol.10, No.2, pp. 9-21, 2020.DOI: 10.5815/ijwmt.2020.02.02 